\title{
UNA NUEVA ESPECIE DE BURSERA (BURSERACEAE) DEL SUR DE MÉXICO
}

\author{
Rosalinda Medina-Lemos \\ Universidad Nacional Autónoma de México, Instituto de Biología, \\ Departamento de Botánica, 3er. Circuito Exterior, \\ Cd. Universitaria, 04510 México, D.F., México. \\ rmedina@ibiologia.unam.mx
}

\section{RESUMEN}

Se describe e ilustra Bursera jerzyi sp. nov., especie arbórea conocida del sur de Oaxaca. Esta especie pertenece a la sección Bullockia y parece estar relacionada con $B$. sarcopoda, de la que se diferencia por la corteza lisa, gris y no exfoliante, las hojas con margen crenado, la escasa pubescencia, así como por la forma y tamaño de los frutos.

Palabras clave: Bullockia, Bursera, México, Oaxaca, taxonomía.

\section{ABSTRACT}

Bursera jerzyi (Burseraceae), a new arborescent species from southern Oaxaca, is described and illustrated. It belongs to sect. Bullockia and seems to be related to $B$. sarcopoda, from which it differs by its smooth, gray, non-exfoliating bark, crenate leaflet margins, sparse pubescence, and the shape and size of the fruits.

Key words: Bullockia, Bursera, Mexico, Oaxaca, taxonomy.

Esta especie fue detectada por D. Daly en material de herbario desde 2008. Al hacer la actualización del listado para las especies de México J. Rzedowski sugirió completar el material con que se contaba para poder describirla. Se tenían ejemplares con hojas y frutos, pero faltaban las flores. A partir de esa fecha y durante tres años se realizaron varias exploraciones a las localidades que estaban registradas 
para obtener las flores pero el esfuerzo fue infructuoso. Dichas búsquedas se hicieron en diferentes épocas del año, y sin embargo no ha sido posible conocer la época de floración; por tal razón se describe sólo con frutos. Las características de las hojas y los frutos no coinciden con las de las otras especies del género (Rzedowski et al., 2005).

Bursera jerzyi Medina sp. nov. Fig. 1.

Arbor dioecia 8-12(14) $\mathrm{m}$ alta, truncus cortice exteriore griseo non exfolianti; folia imparipinnata rhachidi exalata, foliolis (7)9 ellipticis vel ovatis $7-14 \mathrm{~cm}$ longis, $3.5-5 \mathrm{~cm}$ latis, margine crenatis, utrinque minute hispidulis et cum pilis glanduliferis; flores ignoti; infructescentiae laxe paniculatae $10-18 \mathrm{~cm}$ longae pedicellis 5.5-7 cm longis; fructus bivalvati plano-convexi $1.3-1.5 \mathrm{~cm}$ longi, $0.8-1.2 \mathrm{~cm}$ diametro, pyrenae subcirculares 7-9 $\mathrm{mm}$ longae pseudoarillo omnino indutae.

Árbol dioico, de 8 a 12(14) $\mathrm{m}$ de alto, no aromático al estrujarse; tronco hasta de $20 \mathrm{~cm}$ de diámetro, corteza externa gris, lisa, no exfoliante, con numerosas lenticelas, ramas maduras pardo-rojizas, las juveniles hispídulas a glabrescentes cuando maduras; precede a la aparición de las hojas un conjunto de catafilos anchamente deltoides a anchamente triangulares, hasta de $3 \mathrm{~mm}$ de largo, pardo-verdosos, cinéreo-puberulentos por fuera, glabros por dentro, pronto deciduos. Hojas generalmente agrupadas en rosetas en el ápice de las ramas maduras o alternas sobre las ramas de crecimiento nuevo, pecíolos de 8 a 13(15) cm de largo, hispídulos, láminas ovadas en contorno general, de 18 a $40 \mathrm{~cm}$ de largo y 15 a $28 \mathrm{~cm}$ de ancho, por lo general imparipinnadas, raquis sin alas, peciólulos de 0.7 a $1.5 \mathrm{~cm}$ de largo y en el folíolo terminal hasta de $2.4 \mathrm{~cm}$ de largo, folíolos (7)9, elípticos, rara vez ovados, de 7 a $14 \mathrm{~cm}$ de largo y 3.5 a 5(6) cm de ancho, el terminal con frecuencia más ancho que los laterales, ápice agudo a caudado, base de los laterales redondeada y ocasionalmente oblicua o ligeramente cordata, margen crenado regularmente desde el ápice hasta cerca de la base de la lámina y ciliado, membranáceos, a simple vista glabros pero haz y envés hispídulos y con tricomas glandulares, la pubescencia diminuta, principalmente a lo largo de las nervaduras. Infrutescencias de 10 a $18 \mathrm{~cm}$ de largo, en forma de panículas laxas, pedicelos de 5.5 a $7 \mathrm{~cm}$ de largo, frutos 2-valvados, suborbiculares cuando inmaduros y plano-hemisféricos al madurar, de 1.3 a $1.5 \mathrm{~cm}$ largo y 0.8 a $1.2 \mathrm{~cm}$ de diámetro, ápice agudo, glabros y pardos en la madurez, hueso plano-hemisférico en vista dorsi-ventral, de 7 a $9 \mathrm{~mm}$ de largo, cubierto totalmente por un pseudoarilo, blanquecino o amarillento cuando inmaduro. 
Medina-Lemos: Nueva especie de Bursera del sur de México

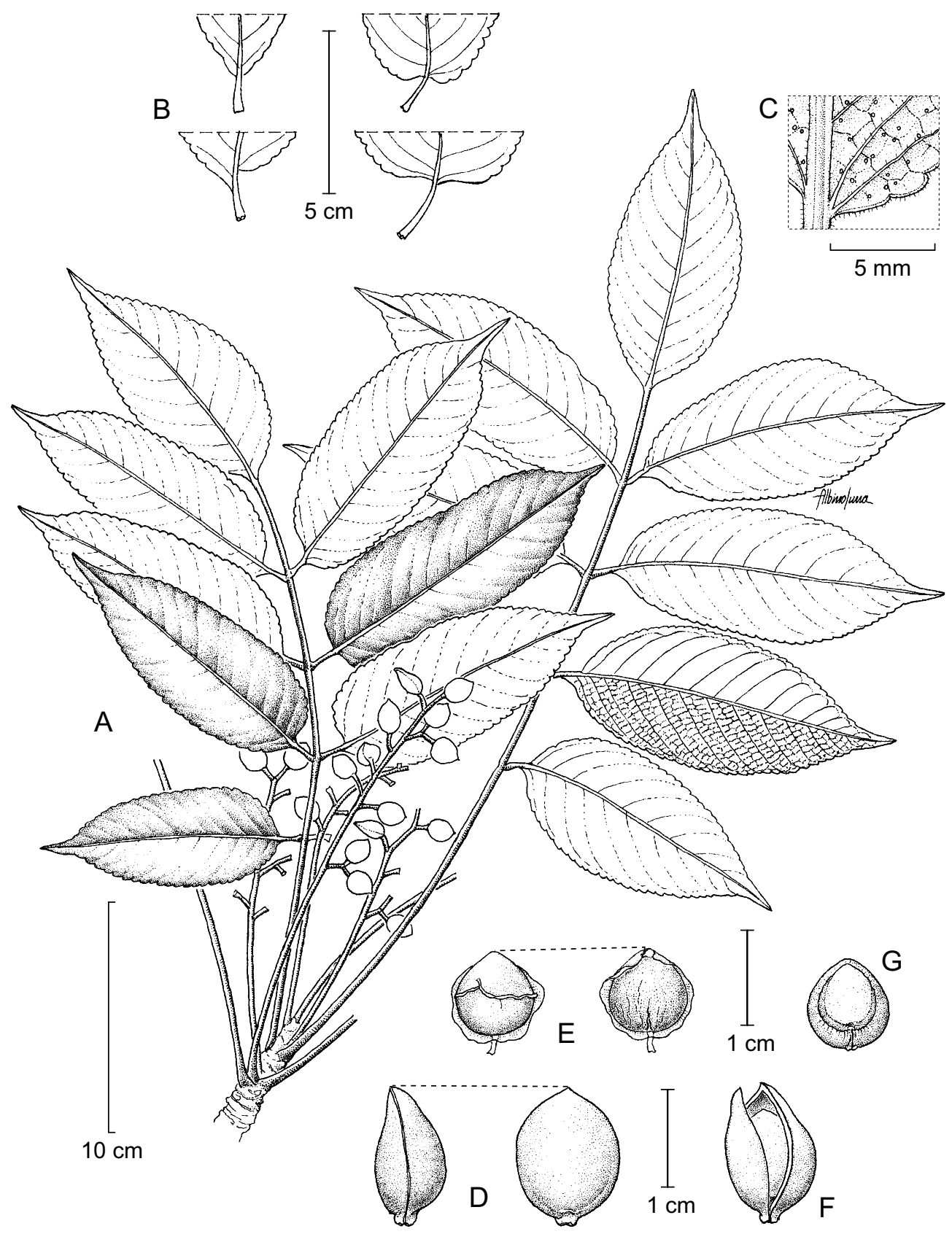

Fig. 1. Bursera jerzyi Medina. A. rama con hojas e infrutescencia; B. base de los folíolos; C. detalle de la pubescencia en el envés; D. fruto, en vista lateral y frontal; E. hueso con pseudoarilo; F. abertura de las valvas; G. hueso sin pseudoarilo. 
TIPO: MÉXICO. Oaxaca: Distrito Pochutla, Santa María Huatulco, Bahía Conejo, 13.VIII.2008, R. Medina Lemos, E. Martínez Salas y Eliezer Cocoletzi Vázquez 4629 (holotipo: MEXU; isotipo: IEB).

Material adicional examinado: México. Oaxaca. Distrito Pochutla: $5 \mathrm{~km}$ al NW de la Bahía de Santa Cruz, 1544'40" N, 96²'10" W, G. Castillo C. et al. 9261 (IEB, MEXU, XAL); 3 km al NO de Santa Cruz, 1544'40" N, 96²'30" W, G. Castillo C. et al. 9490 (MEXU, XAL); km 2 del camino a Santa Cruz Huatulco, al S de la carretera a Salina Cruz, R. Cedillo T. y R. Torres 1562 (MEXU); municipio Santa María Huatulco, $500 \mathrm{~m}$ al oeste del puente Tangolunda, sobre la carretera, M. Elorza C. 6991 (IEB); municipio San Miguel del Puerto, Zimatán, $3 \mathrm{~km}$ LR N del puente por el Chorro, E. Martínez S. et al. 32543 (IEB); municipio Santa María Huatulco, Bahía Conejos, $0.5 \mathrm{~km}$ NE, por un arroyo seco que cruza la carretera costera, S. Salas M. et al. 2351 (IEB); municipio Santa María Huatulco, $1.06 \mathrm{~km}$ después del inicio del parque al basurero municipal, 1545'41.3" N, 969'17.1" W, A. Sánchez-Martínez et al. 913 (IEB, MEXU); municipio Santa María Huatulco, Río Cacalutla hacia la playa, $15^{\circ} 44^{\prime} 45.5^{\prime \prime} \mathrm{N}, 96^{\circ} 10^{\prime} 10.8^{\prime \prime} \mathrm{W}, A$. Sánchez-Martínez et al. 1024 (IEB, MEXU); municipio San Miguel del Puerto, $1.2 \mathrm{~km}$ al norte de la carretera sobre la terracería a Santa Ma. Xadani, 1551'10.2" N, 960'15.7" W, A. Saynes V. et al. 2620 (IEB); municipio Santa María Huatulco, $0.540 \mathrm{~km}$ del entronque a la carretera a playa Cacalutla, $15^{\circ} 44^{\prime} 42.4^{\prime \prime} \mathrm{N}, 96^{\circ} 9^{\prime} 14.2^{\prime \prime}$ W, A. Saynes V. et al. 5624 (IEB, MEXU); $40 \mathrm{~km}$ al SW de Coyol o $20 \mathrm{~km}$ al SW del Río Chacalapa, R. Torres 5429 (MEXU); municipio Santa María Huatulco, por el límite noreste del Parque Nacional Huatulco a $366 \mathrm{~m}$ al SW de la entrada a la Universidad del Mar Campus Huatulco, A. Nava Zafra et al. 1048 (IEB). Distrito Tehuantepec: municipio Santiago Astata, $300 \mathrm{~m}$ al S de la carretera sobre la brecha a El Mojón, 1549'22.2" N, 960'38.8" W, A. Saynes V. et al. 2461 (IEB, MEXU).

Estatus de conservación. La especie se conoce sólo de poblaciones en la costa de los distritos de Pochutla y Tehuantepec en Oaxaca, dentro del área protegida Parque Nacional Huatulco.

Sin embargo, a pesar de ser un área protegida las pocas poblaciones actualmente conocidas se encuentran en riesgo, debido a la destrucción del hábitat, por la cercanía a playas turísticas. La infraestructura que se crea en esta región para albergar cada vez a más turismo y todas las actividades paralelas que favorecen el flujo de visitantes conlleva un drástico deterioro. Un ejemplo de ello es el mal 
manejo de los tiraderos de cascajo y basura en general, así como también la contaminación del manto freático por la descarga de agua residual de las empresas hoteleras.

Habita en el bosque tropical caducifolio, en suelos arcillosos o arenosos, desde el nivel del mar hasta $75 \mathrm{~m}$ de altitud. Convive con Bursera silviae Rzed. \& Calderón, Bursera ovalifolia (Schltdl.) Engl. y Bursera excelsa (Humb., Bonpl. \& Kunth) Engl.

Por el tronco con corteza gris no exfoliante, la presencia de catafilos, el raquis no alado y los frutos 2-valvados, pertenece a la sección Bullockia.

Parece estar relacionada con B. sarcopoda Paul G. Wilson, conocida de Colima a Guerrero (Wilson, 1958). Las diferencias principales entre los dos taxa se señalan en el Cuadro 1 y la Fig. 2.
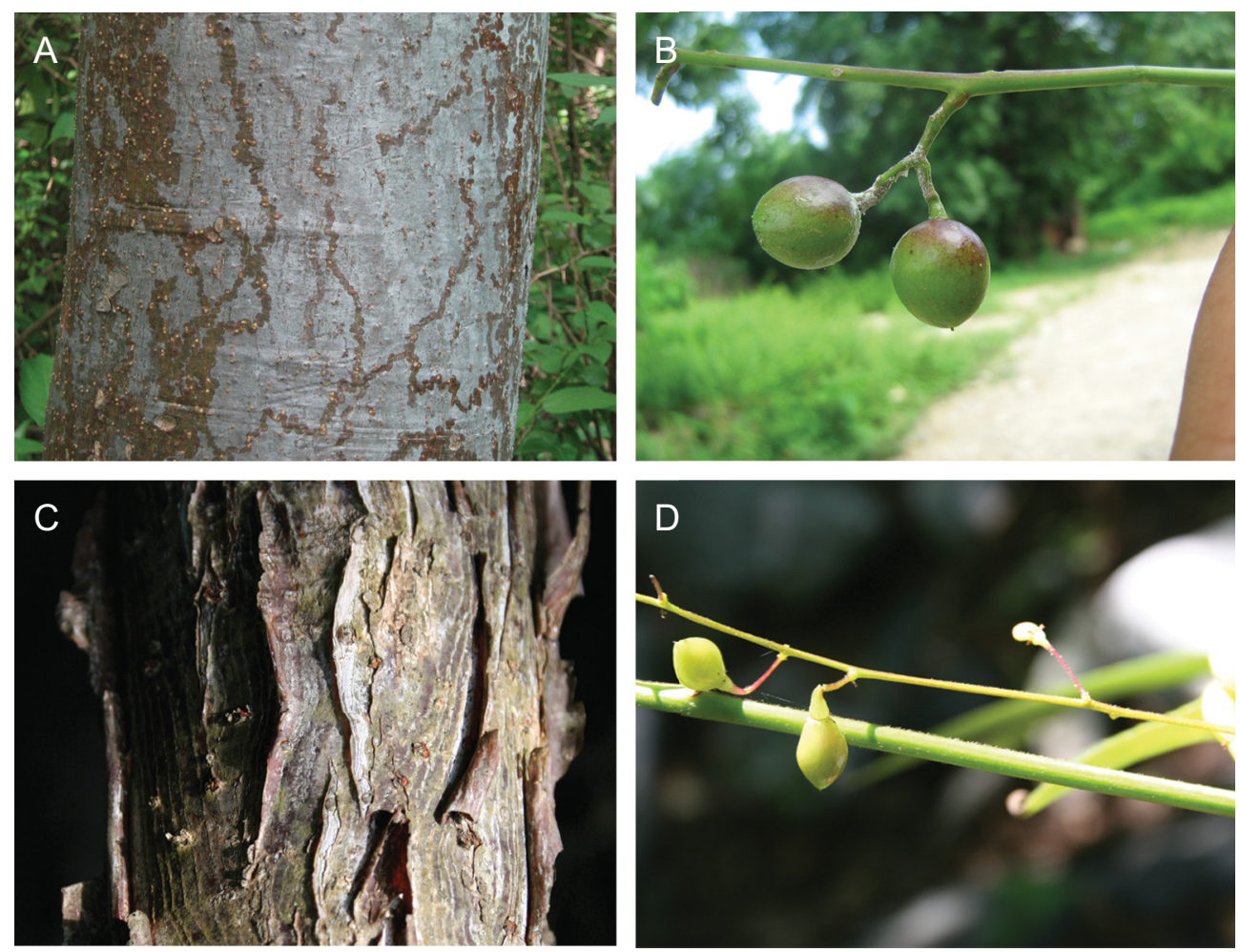

Fig. 2. Bursera jerzyi. A. Corteza gris, no exfoliante; B. Frutos (fotos de E. Martínez Salas). Bursera sarcopoda. C. Corteza roja, exfoliante; D. Frutos (Fotos de Pedro Díaz Maeda). 
Cuadro 1. Caracteres diferenciales entre Bursera sarcopoda y B. jerzyi.

\begin{tabular}{|c|c|c|}
\hline Caracteres & B. sarcopoda & B. jerzyi \\
\hline Corteza & $\begin{array}{l}\text { rojiza, exfoliante en estrías } \\
\text { onduladas verticales }\end{array}$ & gris, no exfoliante y lisa \\
\hline $\begin{array}{l}\text { Pecíolos, peciólulos } \\
\text { y hojas }\end{array}$ & densamente lanosos & $\begin{array}{l}\text { escasamente hispídulos y } \\
\text { con tricomas glandulares } \\
\text { diminutos }\end{array}$ \\
\hline Margen de la hoja & serrado a doblemente serrado & crenado \\
\hline Frutos maduros & elipsoidales con ápice agudo & plano-hemisféricos \\
\hline Hueso & $\begin{array}{l}\text { parcialmente cubierto por el } \\
\text { pseudoarilo, } 1 \text { a } 1.2 \mathrm{~cm} \text { largo } \\
\text { x } 0.4 \text { a } 0.6 \mathrm{~cm}\end{array}$ & $\begin{array}{l}\text { totalmente cubierto por el } \\
\text { pseudoarilo, } 1.3 \text { a } 1.5 \mathrm{~cm} \\
\text { largo y } 0.8 \text { a } 1.2 \mathrm{~cm}\end{array}$ \\
\hline
\end{tabular}

El epíteto hace alusión al nombre de pila del Dr. Jerzy Rzedowski quien ha contribuido ampliamente en el conocimiento de la vegetación de México y en particular en los avances sobre el conocimiento del género Bursera.

\section{AGRADECIMIENTOS}

Se agradece a Albino Luna la elaboración de la ilustración, a Esteban Martínez Salas por el apoyo incondicional en el trabajo de campo y las valiosas fotografías que tomó en campo del nuevo taxon, a Pedro Maeda por las fotografías de B. sarcopoda, al Dr. Fernando Chiang por la traducción del resumen al inglés y por su tiempo para revisar el manuscrito, al Dr. Rzedowski por la diagnosis en latín, así como también a él y la Dra. Graciela Calderón de Rzedowski por la oportunidad de colaborar estos últimos diez años en el conocimiento del género Bursera en México. Así como también a las personas que fungieron como revisores y que mejoraron la presentación de este escrito.

\section{LITERATURA CITADA}

Rzedowski, J., R. Medina-Lemos y G. Calderón de Rzedowski. 2005. Inventario del conocimiento taxonómico, así como de la diversidad y del endemismo regionales de las especies mexicanas de Bursera (Burseraceae). Acta Bot. Mex. 70: 11-85. 
Medina-Lemos: Nueva especie de Bursera del sur de México

Wilson, P. G. 1958. Contributions to the flora of tropical America LXIII: Plantae Hintonianeae XII. Kew Bull. 13: 155-157.

Recibido en mayo de 2012.

Aceptado en enero de 2013. 